\title{
CT angiographic determination of most frequent anatomic variations of coronary arteries in Erbil population
}

\begin{abstract}
Zana Mustafa Mahmood*
Nasih Mohsin Ahmed**

Nali Abdulkader Maaruf*

Abstract

Background and objective: Computed tomography angiography is one of the best methods for knowing the detailed anatomy of coronary arteries and can successfully detect any variation of coronary arteries. Anatomic variations of coronary arteries have not been studied among Erbil population which is mostly inhabited by Kurds. This study was conducted to compare the incidence of the anatomic variations of coronary arteries in Erbil population with international standards.

Methods: Variations of coronary arteries were retrospectively studied by using computed tomography angiography of 412 cases (214 males 198 females) with mean age $51.5 \pm$ 13.5 years (mean \pm SD) who underwent this procedure on suspicion of coronary artery disease. The main indication was chest pain in patients with low to intermediate probability of ischemic heart disease. The present study was carried out in the Department of Radiology, Surgical Specialty Hospital/Cardiac Center in Erbil city.

Results: The right coronary artery was dominant in $86.4 \%$ of cases, while the left main coronary artery was dominant in $10.92 \%$ of cases. Co-dominance was observed in $2.67 \%$ of cases, and Double Ostia of right aortic sinus was observed in $25 \%$ of cases. Long left main coronary artery was observed in $10.68 \%$ of cases. Myocardial bridging was observed in $7.04 \%$ of cases. Other variations were also observed, and their prevalence was recorded.
\end{abstract}

Conclusion: Variations of coronary arteries among Erbil population were recorded and were near to the international standards.

Keywords: CT angiography; Coronary arteries; Double Ostia; Co-dominance.

\section{Introduction}

Computed tomography (CT) coronary angiography is helpful for cardiologists and makes them understand the variations of the anatomy of the coronary arteries and, thus, to alert the cardiologist whenever such a variation is present. This can be of great help to the clinician for planning interventions such as balloon dilatation, stenting or bypass surgery. ${ }^{1}$ For better interpretation of the coronary computed tomography angiography (CTA) correctly, radiologists and cardiologists should know normal anatomy, anatomic variations, and anomalies of the coronary arteries. ${ }^{2}$ There is no clear-cut boundary between normal anatomy and anatomic variations or anomaly, and many variables are to be taken into account. ${ }^{3}$ The coronary arteries are the first vessels that branch from the aorta, normally originating below the junction between the bulbs and the ascending aorta, that is, at the sinotubular junction. The coronary orifices are located in the center of the corresponding aortic sinuses and slightly above the free margin of the cusp. ${ }^{4}$ The left coronary artery arises commonly by a single orifice situated at the level of the left aortic sinus. It has a variable length, but it is not usually more than a few millimeters. It passes behind the outlet of the right ventricle and below the left atrial appendage. Normally, the left coronary artery divides into the anterior

* Department of Anatomy and Histology, College of Medicine, Hawler Medical University, Erbil, I raq.

** Department of Radiology, Surgical Specialty Hospital, Cardiac Center, Erbil, I raq. 
https:/ / doi.org/ 10.15218/ zjms.2019.003

interventricular artery and the circumflex artery. ${ }^{4}$ It is known that the left coronary artery has a wider caliber than the right one and is more preponderant, i.e., its distribution area is larger. This artery has the circumflex branch that, in its turn, gives several atrial and ventricular branches and the most constant is the marginal branch that passes down the left margin of the heart. ${ }^{5}$ It arises from a single orifice in the right aortic sinus. In the first millimeters, it is submerged in the adipose tissue of the epicardium below the right atrial appendix (the Rindfleisch fold). It passes above the right atrioventricular groove, and commonly reaches the posterior interventricular groove, frequently passing above the crux cordis. ${ }^{4}$ There are geographical differences in the coronary artery variations, which might have a genetic basis and information about these variations are vital to a cardiac surgeon during management of patients. ${ }^{6}$ This study aimed to determine the most frequent variations in the origin, course, and branching pattern of the coronary arteries among the Erbil population, by using CT coronary angiography retrospectively. Also to study gender association with these variations.

\section{Methods}

\section{Design and setting}

A retrospective study was conducted on consecutive patients who required coronary CTA from January 2012 to September 2016 in the Department of Radiology, Surgical Specialty Hospital/Cardiac Center in Erbil, Kurdistan region, Iraq. The study was approved by the ethics and scientific committees of the College of Medicine, Hawler Medical University.

\section{Study population}

A total of 412 cases including 214 males and 198 females with a mean ( \pm SD) age of $51.5 \pm 13.5$ years were included in the study. The main criteria to include patients in the study included:

1. Chest pain in patients with mild to moderate probability of coronary artery disease.
2. Asymptomatic high-risk patients suspected of having coronary artery disease.

3. Patients who underwent CT angiography for the evaluation of cardiac masses.

4. Preoperative evaluation of patients with non-cardiac surgery and patients with valvular heart disease in need of a valve replacement.

\section{Exclusion criteria}

1. Patients with coronary artery stent.

2. A patient who underwent by-pass grafts.

3. Heavily calcified coronary arteries in whom Ca.score is more than 400 where the anatomy of coronary arteries not visualized well.

4. Patients with prosthetic heart valves.

5. Poor image quality due to severe technical artifacts.

6. Patients with irregular rhythm and high heart rate.

\section{Cardiac CT scanning protocol}

The patients were evaluated by cardiac CT scanning using a 256-slice MSCT (Brilliant ICT, Philips Medical System). Before scanning, blood pressure and heart rate were measured, sublingual nitroglycerin was administered to all of the patients, and those with an initial heart rate above 65 beats per minute were administered beta-blockers in the absence of contraindications. Thereafter, unenhanced electrocardiographically gated CT images were acquired to calculate ca. score before coronary angiography, which were prospectively triggered at $75 \%$ of the $\mathrm{RR}$ interval and obtained under the following parameters: collimation, $4 \times 3.0 \mathrm{~mm}$; gantry rotation time, $0.25 \mathrm{sec}$; tube voltage, $120 \mathrm{kV}$; and tube current, $300 \mathrm{~mA}$. The patients' renal function were preassessed by serum creatinine level, and they were asked to abstain from caffeine, tobacco, and other stimulants for 24 hours before the scan. Intravenous access was obtained in the ante-cubital fossa with a 20 $\mathrm{G}$ cannula. $70-75 \mathrm{mLs}$ of non-ionic contrast containing $350 \mathrm{mg}$ of lodine $/ \mathrm{mL}$ was injected at a rate of $5-6 \mathrm{mls} / \mathrm{sec}$ followed by $40 \mathrm{ml}$ of normal saline. The scan was 
https:/ / doi.org/ 10.15218/ zjms.2019.003

obtained in a single breath hold from the pulmonary hilum to the base of the heart. The data was retrospectively gated and reconstructed at $40 \%$ and $78 \%$ of the $\mathrm{RR}$ interval. Further reconstructions were carried out as required to assess individual segments. Data were transferred to a workstation for analysis. The obtained data were statistically analyzed by using the statistical package for the social sciences (version 21). For descriptive analysis, we used percentage and frequencies, and for further statistical analysis, we used Chi-square test and P-value less than 0.05

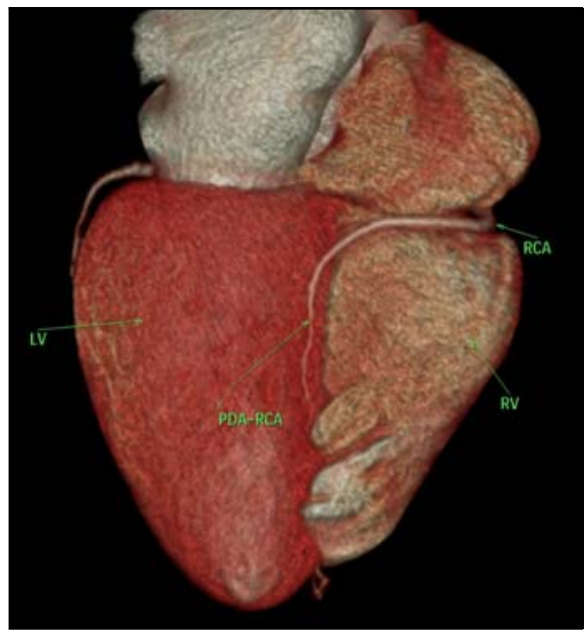

Figure 1: Right dominance

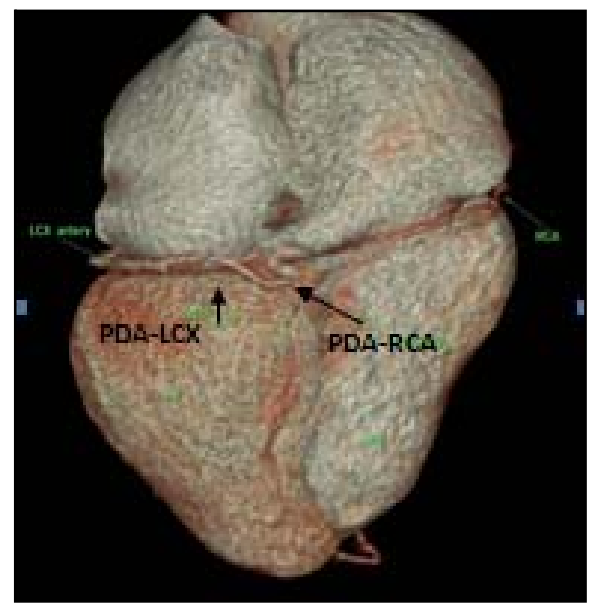

Figure 3: Co dominance was considered statistically significant.

\section{Results}

Apart from coronary dominance and origin of SA nodal artery the overall anatomic variations were present in $65.53 \%$ of cases. The results of the present study showed that in $(86.4 \%)$ of cases, the Right coronary artery (RCA) was dominant; (Figure 1). The left coronary artery was dominant in $10.92 \%$ of cases (Figure 2), and co-dominance was observed in $2.67 \%$ of cases (Figures 3 and 4).

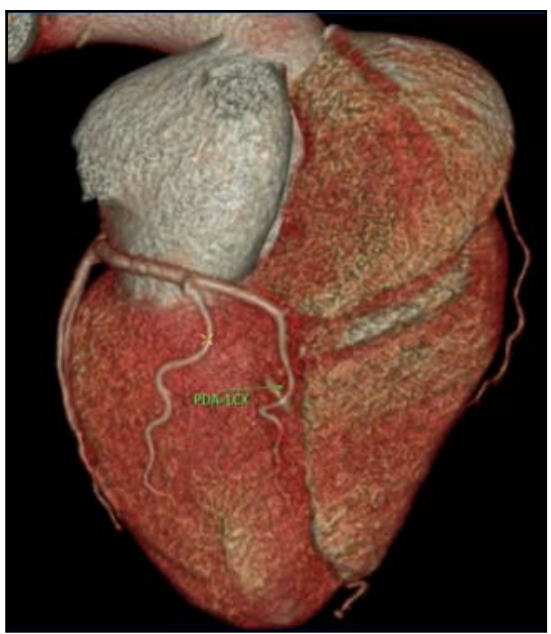

Figure 2: Left dominance

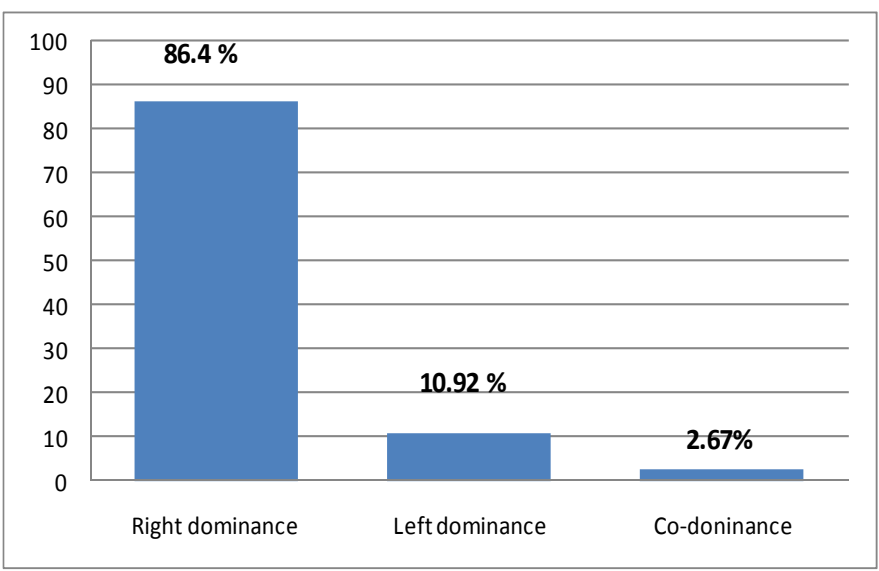

Figure 4: Percentage of types of coronary dominance 
https:// doi.org/ 10.15218/ zjms.2019.003

In $(35.44 \%)$, the left main coronary artery (LMCA) trifurcated to include a third vessel between the left anterior descending artery (LAD) and Cx, termed median artery or ramus intermedius (Figure 5). The right conus artery took its origin from the right aortic sinus by a separate ostium in $(25 \%)$ of cases (Figure 6 ); while in $(0.49 \%)$ of cases, two conus arteries took their origin with separate ostia from the right aortic sinus. The sinus node artery took its origin from the RCA as its second branch in $(82.6 \%)$ of cases, from the $\mathrm{Cx}$ in $(16.77 \%)$, and the LMCA in $(0.49 \%)$ of cases. In $(0.24 \%)$, the sinus node artery originated from the right aortic sinus with a separate

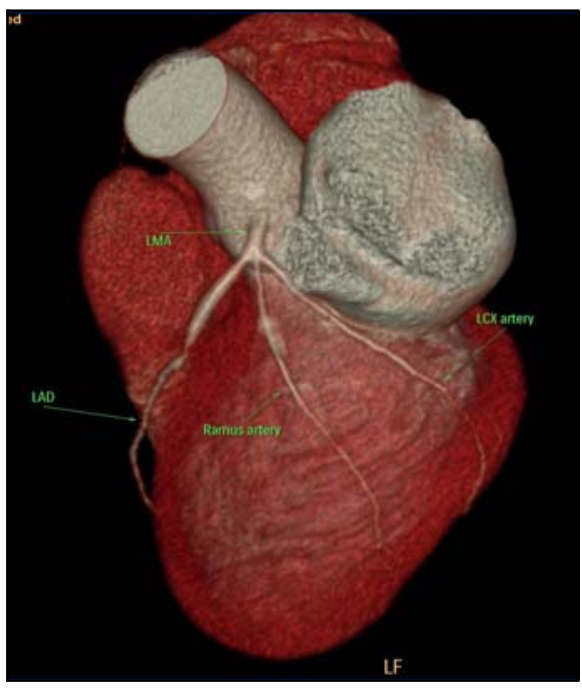

Figure 5: Median artery (Ramus intermedius).

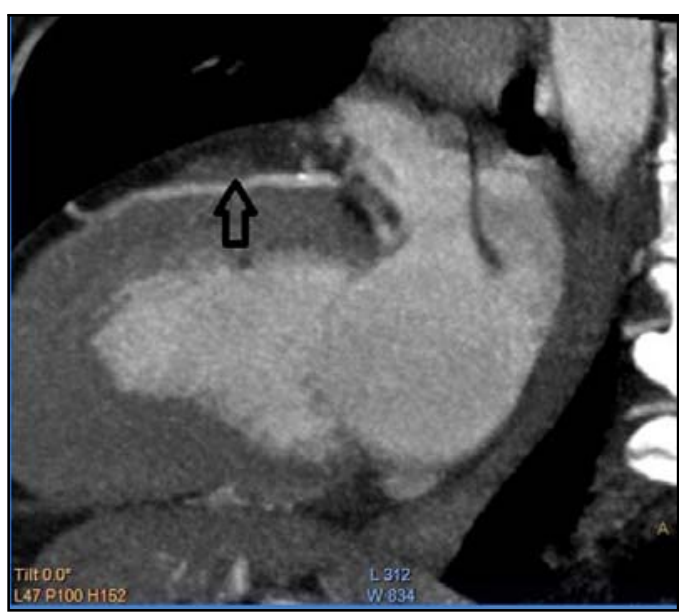

Figure 7: Myocardiac bridge (black arrow) ostium. High origin of the RCA and LMCA were observed in $13(3.16 \%)$ and five cases $(1.21 \%)$, respectively. The RCA divided into two arteries just after originating from its ostium in the right aortic sinus (termed duplication of RCA) in $(2.91 \%)$ of cases. In $(0.49 \%)$ of cases, the Cx artery was absent, and the RCA flowed in the posterior aspect of the left atrioventricular $(A-V)$ groove through the track of the Cx. Myocardial bridging was observed in $(7.04 \%)$ of cases (Figure 7 ), and it was mostly found in the anterior descending artery. In (8.25\%) of cases left main coronary artery was less than $5 \mathrm{~mm}$ and considered as being short (Figure 8).

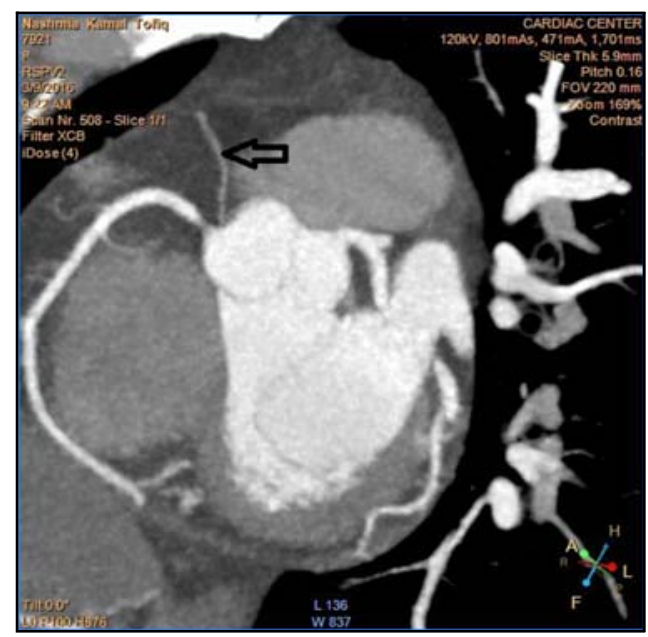

Figuer 6: Separate ostium of right conus artery from right aortic sinus (Black arrow)

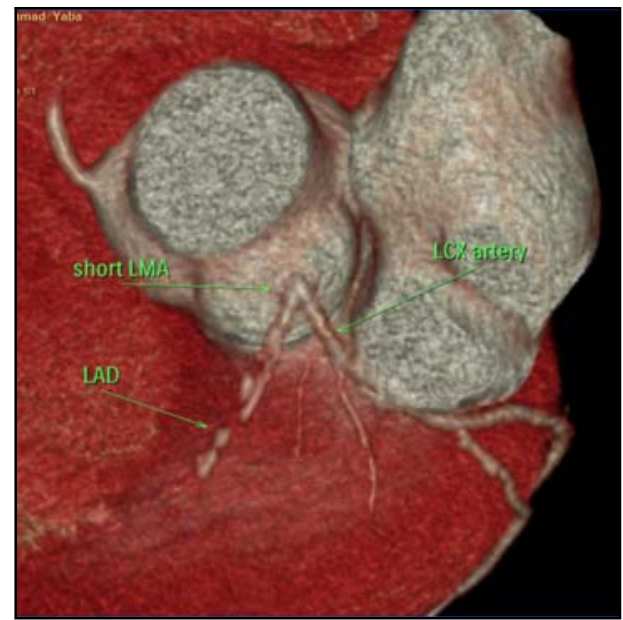

Figure 8: Short LMCA 
https:/ / doi.org/ 10.15218/ zjms.2019.003

In $10.68 \%$ of cases it was more than 15 $\mathrm{mm}$, and it was considered as being long (Figure 9). Figure 10 illustrates the frequency of the most common variations. Regarding the gender distribution, we observed that all common variations were more frequent in males except the duplication of RCA, which was more frequent in females and absence of circumflex and triple ostium of the right aortic sinus, which was equally frequent in both sexes (Figure 10). Chi-square test showed that there was a statistically significant association between three types of variations and gender. The variations were median artery and double ostium of the right aortic sinus, which were more frequent in males, and the duplication of RCA, which was more frequent in females (Table 1 and Figure 10).

Table 1: Association of gender with coronary artery variation types.

\begin{tabular}{|c|c|c|c|c|c|}
\hline \multicolumn{3}{|c|}{ Variations } & \multirow{2}{*}{$\begin{array}{c}\text { Male } \\
94\end{array}$} & \multirow{2}{*}{$\begin{array}{c}\text { Female } \\
52\end{array}$} & \multirow{3}{*}{$\begin{array}{l}\boldsymbol{P} \text { value } \\
<0.001\end{array}$} \\
\hline 1 & Median artery (Ramus intermedius) & Yes & & & \\
\hline & & No & 120 & 146 & \\
\hline \multirow[t]{2}{*}{2} & The double ostium of right aortic sinus (right & Yes & 65 & 38 & \multirow{2}{*}{0.009} \\
\hline & conus+ RCA) & No & 149 & 160 & \\
\hline \multirow[t]{2}{*}{3} & Long LMCA & Yes & 25 & 19 & \multirow{2}{*}{0.493} \\
\hline & & No & 189 & 179 & \\
\hline \multirow[t]{2}{*}{4} & Short LMCA & Yes & 18 & 16 & \multirow{2}{*}{0.903} \\
\hline & & No & 196 & 182 & \\
\hline \multirow[t]{2}{*}{5} & Myocardial bridging & Yes & 16 & 13 & \multirow{2}{*}{0.718} \\
\hline & & No & 198 & 185 & \\
\hline \multirow[t]{2}{*}{6} & High origin of RCA & Yes & 10 & 3 & \multirow{2}{*}{0.067} \\
\hline & & No & 204 & 195 & \\
\hline \multirow[t]{2}{*}{7} & Duplication of RCA & Yes & 1 & 11 & \multirow{2}{*}{0.002} \\
\hline & & No & 213 & 187 & \\
\hline \multirow[t]{2}{*}{8} & High origin of LMCA & Yes & 3 & 2 & \multirow{2}{*}{0.717} \\
\hline & & No & 211 & 196 & \\
\hline \multirow[t]{2}{*}{9} & Triple ostium of right aortic sinus ( 2 conus arteries & Yes & 1 & 1 & \multirow{2}{*}{0.956} \\
\hline & and RCA) & No & 213 & 197 & \\
\hline \multirow[t]{2}{*}{10} & Absence of circumflex & Yes & 1 & 1 & \multirow{2}{*}{0.956} \\
\hline & & No & 213 & 197 & \\
\hline
\end{tabular}

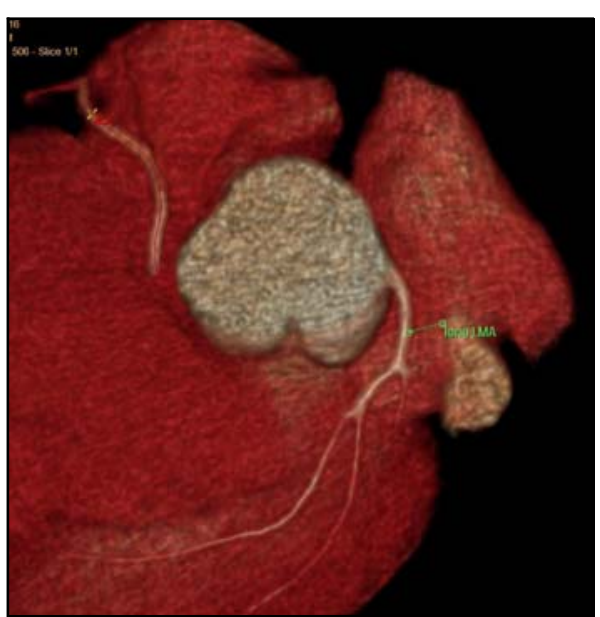

Figure 9: Long LMCA

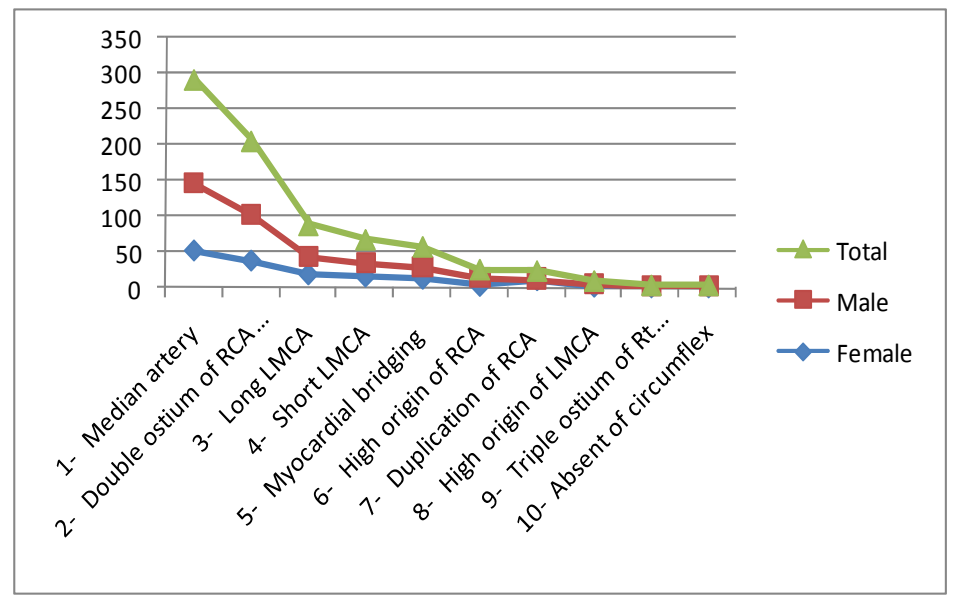

Figure 10: Frequency of most common variations in the total sample, in the males and in the females. 
We used a Chi-square test for finding the association of the frequency of the ive most common variations with the type of coronary dominance, and there was no statistically significant association
(Table 2). There is a significant statistical association between the presence of the median artery with the length of LMCA $(P<0.001)$ as shown in Table 3.

Table 2: Association of coronary dominance with some common anatomic variations of coronary arteries.

\begin{tabular}{lccccc}
\hline Variations & & $\begin{array}{c}\text { Right } \\
\text { Dominance }\end{array}$ & $\begin{array}{c}\text { Left } \\
\text { Dominance }\end{array}$ & Co-Dominance & $P$ value \\
\hline $\begin{array}{l}\text { Median artery } \\
\text { (Ramus intermedius) }\end{array}$ & Yes & 122 & 19 & 5 & 0.449 \\
& No & 234 & 26 & 6 & \\
2 The double ostium of right \\
$\begin{array}{l}\text { aortic sinus } \\
\text { (right conus+ RCA) }\end{array}$ & Yes & 94 & 8 & 1 & 0.211 \\
& No & 262 & 37 & 10 & \\
3 Long LMCA & Yes & 40 & 3 & 1 & 0.636 \\
& No & 316 & 42 & 10 & \\
4 Short LMCA & Yes & 27 & 6 & 1 & 0.416 \\
& No & 329 & 39 & 10 & 0.202 \\
5 Myocardial bridging & Yes & 22 & 6 & 1 & \\
\hline
\end{tabular}

Table 3: Association of the presence of the median artery with the length of LMCA.

\begin{tabular}{lcccc} 
LMCA types & Median artery Yes & Median artery No & Total & $P$ value \\
\hline Normal LMCA & 117 & 217 & 334 & \\
Long LMCA & 25 & 19 & 44 & $<0.001$ \\
Short LMCA & 4 & 30 & 34 &
\end{tabular}


https:/ / doi.org/ 10.15218/ zjms.2019.003

\section{Discussion}

The complex anatomy of the coronary arteries can precisely be studied by CTA. This modality is very useful in detecting coronary artery variations and is superior to conventional coronary angiography in their diagnosis. ${ }^{2}$ There is no clear-cut boundary between variation and anomaly, but as a general rule, if the deviation from the popularity causes symptoms and harm to the subject, it may be regarded as the anomaly rather than a normal variation. Coronary dominance should be recorded in every CTA report. If the diaphragmatic surface of the heart is supplied by the right coronary artery, it is considered as right dominance. If it is supplied mostly by the left coronary artery, it is considered as left dominance. In the present study, $86.4 \%$ of cases had right dominance, $10.92 \mathrm{had}$ left dominance, and $2.67 \%$ were co-dominant that is to say both arteries share in the supply of diaphragmatic surface. This result is comparable with previous literature results as it was shown by Jyoti Kulkarni in $79 \%$, the right coronary artery was dominant. $^{7}$ The LMCA length was regarded as being long if it was more than $15 \mathrm{~mm}$ which was present in $10.68 \%$ of cases. In literature, this long LMCA was $11.5 \%-18 \%$ as it was reported by Helwing 1967 which is near to the present study results ${ }^{4}$. In $8.25 \%$ it was less than $5 \mathrm{~mm}$, and this short LMCA has significance during coronary angiography. If the artery is short it may be clinically relevant, especially when a perioperative coronary perfusion or a coronary graph is performed. An incomplete image of the area of distribution of the left coronary artery may be seen on introducing the catheter into only one of the terminal branches, and the other does not then show opacification. ${ }^{8}$ In $35.44 \%$ of cases, the LMCA is trifurcated into three vessels instead of dividing into two arteries (Cx and LAD). The intermediate artery is termed as the median artery or ramus intermedius. This incidence is similar to another study $(33 \%)$ as it was reported by Pinar et al. 2009. ${ }^{2}$ In another study by
Fazllogullar et al., they observed that the LMCA gave rise to two branches (called bifurcation) in $46 \%$ of cases, three branches (called trifurcation) in $44 \%$ and four branches (called quadrifurcation) in $10 \%$ of the cases. The median artery (ramus intermedius) was noticed in 27 cases out of $50 .^{9}$ The popularity trifurcation rates suggest that the normal division of the LCA into two branches should be reconsidered. The caliber of the median artery may be occasionally similar to that of the anterior interventricular artery or greater than that of the circumflex artery. For this reason, Levin (1983) states that, unlike certain hemodynamics, we should not focus our angiographic examination solely on the search for lesions in the anterior interventricular and circumflex arteries since the involvement of the median artery may depending on its distribution, be as dangerous as the involvement of the two arteries. ${ }^{10}$ In agreement with our results, Sunil Kini et al. stated that the most frequent variation in left coronary artery anatomy is the presence of a trifurcation of the LMCA (presence of median artery). ${ }^{11}$ The high take-off of the right coronary artery (above sinotubular junction) was observed in $3.16 \%$ of cases, which was more prevalent than what was reported by Pinar et al. which was $0.7 \%{ }^{2}$ The left coronary orifice high take-off prevalence was $1.21 \%$, which was less than a study conducted on Indian people $(2 \%)$ by Tomar et al. ${ }^{12} \mathrm{~A}$ high left coronary orifice is commonly at a greater risk during surgery or coronary intervention because it is usually associated with a long left coronary artery and this injury may be due to low clamping of the aorta or due to the incision in the aorta wall during valvular replacement. ${ }^{4}$ Most haemodynamists agree that high and low coronary orifices represent an added difficulty in coronary angiography. ${ }^{13}$ Double ostia of the right coronary artery (conus artery and right coronary artery) were common (25\% of cases). Which is near to what was reported by Pinar et al. $(22 \%){ }^{2}$ Triple ostia of the 
right coronary artery ( 2 conus arteries and the right coronary artery were found in 2 cases $0.49 \%$. Presence of multiple ostia in the right sinus Valsalva may also lead to surgical problems in cases requiring right ventriculotomy for the ventricular septal defect, or pulmonary stenosis. ${ }^{2}$ Multiple coronary ostia are probably one of the most frequent variations of the normal coronary artery anatomy. ${ }^{14}$ The left coronary artery is said to arise from a single ostium virtually always according to (James, 196I). The place of the ostium can vary from the cusp margin to a position one to two $\mathrm{cm}$ above the margin of the sinus. In the present study in agreement with (James, 1961), no any case in the sample reported having multiple ostia in the left aortic sinus. ${ }^{14}$ The intramyocardial course of coronary arteries is referred to as myocardial bridging, which was found in $7.04 \%$ of cases. Sometimes, the contraction of myocardial bridges may reduce the caliber of the artery by more than $75 \%$, and so in situations requiring a substantial oxygen supply to the myocardial cells. The electrocardiogram may present anomalies compatible with ischemia and lactate production. This is the basis for the hypothesis that the myocardial bridges may be the cause of myocardial ischemia. ${ }^{15}$ The myocardial bridging was most commonly found in the anterior descending artery, and this result was in agreement with Catarina et al. ${ }^{16}$ There is very little literature on gender difference in coronary artery variations. In the present study, the median artery and the double ostium of right aortic sinus were more frequent in males, and the association was statistically significant. This finding does not agree with (Tomar et al.) who found that the median artery was not significantly different in males and females. ${ }^{12}$ In the present study, myocardial bridging was more common in males (with no statistically significant association). This difference was found to be significant in another study done by (Yuksel et al.) who found that myocardial bridging was more common in males and the difference was statistically significant. ${ }^{17}$ There was no statistically significant association between coronary dominance and common anatomic variations of coronary arteries in the present study. As far as the theory of Ogden $\mathrm{J}$. there is a dual origin of coronary arteries distal and proximal. The first one to develop is the distal portion. It is composed of a retiform vascular network, which is similar to the capillary network that is created in the other parts of the body. This network develops in the atrioventricular and interventricular sulci and forms a complete ring around the developing vessels, and communicates with the heart chambers and the great vessels. Further development of some vessels and regression of others; the final coronary pattern develops. This theory explains the variations of coronary vasculature. ${ }^{7}$

\section{Conclusion}

The common variations of coronary arteries among the Erbil population are comparable with and near to international standards. Moreover, it is important for the cardiologist or cardiac surgeon to be familiar with these variations prior to undergoing any procedure. We recommend doing similar studies in other cities of Kurdistan region and with larger sample sizes to measure the frequency of these variations in the Kurdish population.

\section{Competing interests}

The authors declare that they have no competing interests.

\section{References}

1. Rahalkar AM, Rahalkar MD. Pictorial essay: coronary artery variants and anomalies. Indian J Radiol Imaging 2009; 19(1):49-53.

2. Pinar K, Elif E, Cansu Ö, Uğur K. Anatomic variations and anomalies of the coronary arteries: 64-slice CT angiographic appearance. Diagn Interv Radiol 2009; 15:27583.

3. Horia M. Coronary arterial anomalies and variations. MEDICA J Clin Med 2006; 1(1):3848.

4. Reig V. Anatomical variations of the coronary arteries: The most frequent variations. Eur J Anat 2003; 7(1):29-41. 
5. ALVES N. Origin of the circumflex branch of the coronary artery. A case report. Int $\mathrm{J}$ Morphol 2008; 26(1):39-41.

6. Qazi WU, Nazish WF, Shemaila SM, Khadija Q. Variation in the Number and Location of Coronary Ostia - A Cadaveric Study. Int J Pathol 2015; 13(3):95-100.

7. Jyoti K, Lopa M. Study of angiographic anatomy of right coronary artery. JDMS 2012; 5(4):2-10.

8. Vlodaver Z, Amplatz K, Burchell HB, Edwards JE. Coronary heart disease. Clinical, angiographic and pathologic profiles. SV New York 1976; 9(4):123-58.

9. Fazliogullari Z, Karabulut AK, Unver Dogan N, Uysal II. Coronary artery variations and median artery in Turkish cadaver hearts. Singapore Med J 2010; 51(10):775.

10. Roberts WC, Silver MA, Sapala JC. Intussusception of a coronary artery associated with sudden death in a college football player. Am J Cardiol 1986; 5(7):179-80.

11. Sunil K, Kostaki G B, Leroy W. Normal and Variant Coronary Arterial and Venous Anatomy on High-Resolution CT Angiography. AJR 2007; 188:1665-74

12. Tomar S, Aga P, Sharma PK, Manik P, Srivastava AK. Normal and variant anomaly of left coronary artery: 64 slice multidetector computed tomography coronary angiographic depictation in north India population. IJSRP 2013; $3(8): 1-17$.

13. Greenberg MA, Fisch BG, Spindola-Franco $H$. Congenital anomalies of the coronary arteries. Classification and Significance. Radiol Clin N Amer 1989; 27:1127-46.

14. Roy PR, Saunders A, Sowton GE. Review of variations in origin of left circumflex coronary artery. BHJ 1975; 37:287-92.

15. Noble J, Bourassa MG, Petitclerc R, Dyrda I. Myocardial bridging and milking effect of the left anterior descending coronary artery: Normal variant or obstruction? Am J Cardiol 1976; 37:993 -9 .

16. Catarina AO, Paula M, Susana $P$, Basso RP Congenital Coronary Variants and Anomalies: Prevalence in Cardiovascular Multislice Computed Tomography Studies in a Single Center. Open Journal of Radiology 2014; 4:16317.

17. Yuksel A, Huseyin UY, Alparslan B, Muharrem N, Aydin N, Taner U, et al. Gender Difference in the atypes and Frequency of Coronary Artery Anomalies. Tohoku J Exp Med 2011; 225:23947. 